\title{
Potential function of CTLA-4 in the tumourigenic capacity of melanoma stem cells
}

\author{
BINGYU ZHANG ${ }^{1}$, JIANZHONG DANG ${ }^{2}$, DIANDIAN BA ${ }^{1}$, CENCEN WANG $^{1}$, JUAN HAN ${ }^{1}$ and FANG ZHENG ${ }^{1}$ \\ ${ }^{1}$ Department of Paediatrics, Union Hospital, Tongji Medical College, Huazhong University of Science and Technology, \\ Wuhan, Hubei 430022; ${ }^{2}$ Department of Geriatrics, Renmin Hospital of Wuhan University, Wuhan, Hubei 430060, P.R. China
}

Received November 29, 2016; Accepted November 10, 2017

DOI: $10.3892 / \mathrm{ol} .2018 .9354$

\begin{abstract}
Extensive clinical evidence supports that cytotoxic T lymphocyte antigen-4 (CTLA-4) is expressed in a variety of human malignant tumour cells in addition to $\mathrm{T}$ cells. In certain types of cancer, the overexpression of CTLA-4 is associated with poor patient prognosis. However, few studies have demonstrated the effects of tumour-intrinsic CTLA-4 in cancer stem cells, including melanoma stem cells (MSCs). In the present study, it was demonstrated that melanoma cell-intrinsic CTLA-4 induced tumour cell proliferation in vitro and suppressed tumour cell apoptosis. Furthermore, CTLA-4 was expressed in aldehyde dehydrogenase (ALDH) ${ }^{+}$ MSCs. CTLA-4 inhibited MSCs proliferation in vitro by blocking antibodies and significantly downregulated ALDH1A1, ALDH1A3 and ALDH2 mRNA expression $(\mathrm{P}<0.01)$. Functionally, blocking CTLA-4 in melanoma cell lines suppressed the properties of stem-like cells, including ALDH activity and significantly suppressed the ability of these cells to form spheres in vitro $(\mathrm{P}<0.05)$. In addition, the blocking of CTLA-4 in melanoma cells suppressed the properties of stem-like cells in vivo, including the capacity for tumourigenesis. The presence of residual $\mathrm{ALDH}^{+} \mathrm{MSCs}$ within the tumour was observed, and the blocking CTLA-4 significantly decreased the number of residual $\mathrm{ALDH}^{+}$ MSCs in vivo $(\mathrm{P}<0.01)$. Altogether, these results indicate the identification of a novel mechanism underlying melanoma progression in the present study and that CTLA-4-targeted therapy may benefit candidate CTLA-4-targeted therapy by
\end{abstract}

Correspondence to: Dr Fang Zheng, Department of Paediatrics, Union Hospital, Tongji Medical College, Huazhong University of Science and Technology, 1277 JieFang Avenue, Wuhan, Hubei 430022, P.R. China

E-mail: zhengfang0883@163.com

Abbreviations: MSCs, melanoma stem cells; CTLA-4, cytotoxic T lymphocyte antigen-4; ALDH, aldehyde dehydrogenase

Key words: cytotoxic T lymphocyte antigen-4, melanoma, cancer stem cells, tumourigenesis, apoptosis improving the long-term outcome for patients with advanced stages of melanoma.

\section{Introduction}

Melanoma, one of the most aggressive cancer types, accounts for $<5 \%$ of all skin cancer types but is responsible for the majority of incidences of skin cancer-associated mortality globally (1). It has been hypothesized that melanoma stem cells (MSCs) possess the capacity for self-renewal, differentiation, chemoresistance and immune evasion (2,3). Thus, understanding the mechanism underlying melanoma progression may contribute to the targeted killing of MSCs, which may improve the efficacy of anticancer therapies and decrease the risk of relapse and progression.

Cytotoxic T lymphocyte antigen-4 (CTLA-4) is a co-stimulatory molecule that is expressed on activated $\mathrm{T}$ cells and competes with T-cell-specific surface glycoprotein CD28 for binding to B7 and inhibits T-cell proliferation, activation and immune response $(4,5)$. CTLA-4 is also expressed on regulatory $\mathrm{T}$ cells with immunosuppressive properties $(6,7)$. Studies have demonstrated that the human CTLA-4 blocking antibody, ipilimumab, which was approved by the United States Food and Drug Administration for the monotherapy of advanced melanoma in March 2011, has a response rate of 11\% and prolongs overall survival in $22 \%$ of patients $(8,9)$. As a subset of patients treated with CTLA-4 blockade, ipilimumab, exhibited an objective clinical response, it may be advantageous to investigate the molecular mechanisms underlying CTLA-4 anti-tumour activity and identify patients who are most likely to respond.

CTLA-4 has also been implicated to serve functions in neoplastic cells, including B cell chronic lymphocytic leukaemia (10), non-Hodgkin's lymphoma (11), breast cancer (12), lung cancer (13), melanoma (14), gastric cancer (15), colorectal cancer (15), cervical cancer (16) and oesophageal carcinoma (17). Prior studies indicated that CTLA-4 expression in tumour cells is associated with poor prognosis $(12,17)$. CTLA-4 engagement with B7 ligands induces tumour cell death through apoptosis (18), and ipilimumab triggers effector lymphocytes and tumour necrosis factor release to directly eliminate CTLA- $4^{+}$melanomas (14). These studies have indicated the potential function of CTLA-4 in tumour cells. Furthermore, CTLA-4 was overexpressed in allogeneic 
mesenchymal stem cells, which resulted in the suppression of the immune response and the promotion of osteogenic differentiation (19). It has been indicated that CTLA-4 is involved in regulating the biological characteristics of stem cells (19). Extensive clinical evidence supports the use of CTLA-4 blockade against advanced melanoma $(8,9)$. Nevertheless, the involvement of the CTLA-4 molecule in regulating the tumourigenic capacity of MSCs is not well understood.

In the present study, the expression of CTLA-4 in MSCs in melanoma cell lines were examined and the function of CTLA-4 in maintaining the characteristics of MSCs were investigated. The results demonstrated that the CTLA-4 molecule participated in the tumourspheres proliferation in vitro and apoptosis of melanoma cells. Furthermore, CTLA-4 was expressed in MSCs and was involved in enhancing the aldehyde dehydrogenase (ALDH) activity and tumourigenic capacity of MSCs.

\section{Materials and methods}

Cell culture. The mouse melanoma B16-F0 and B16-F1 cell lines (American Type Culture Collection, Manassas, VA, USA) were maintained in RPMI-1640 (Gibco; Thermo Fisher Scientific, Inc., Waltham, MA, USA) containing $10 \%$ foetal bovine serum (ScienCell Research Laboratories, Inc., San Diego, USA), $100 \mathrm{U} / \mathrm{ml}$ penicillin (Gibco; Thermo Fisher Scientific, Inc.) and $100 \mu \mathrm{g} / \mathrm{ml}$ streptomycin (Gibco; Thermo Fisher Scientific, Inc.). Cells were cultured at $37^{\circ} \mathrm{C}$, $95 \%$ humidity and $5 \% \mathrm{CO}_{2}$.

Flow cytometry. To determine the expression of CTLA-4 in melanoma cells, B16-F0 and B16-F1 cells were surface stained for CTLA-4. A total of $1 \times 10^{6}$ cells/ml B16-F0 or B16-F1 cells were suspended in PBS at room temperature. To one tube of cells, $5 \mu$ l anti-CTLA-4 antibody (cat. no. 553720; dilution 1:200PE; BD Pharmingen; BD Biosciences) was added, and to one tube of cells $5 \mu \mathrm{l}$ immunoglobulin $\mathrm{G}$ isotype-matched control (BD Pharmingen; BD Biosciences) was added as a negative control at room temperature. The tubes were incubated for $30 \mathrm{~min}$ at $4^{\circ} \mathrm{C}$. Following incubation, centrifugation was performed at $4^{\circ} \mathrm{C}$ for $10 \mathrm{~min}$ at $12,000 \mathrm{x} \mathrm{g}$ and the pellets were re-suspended with $500 \mu 1$ of Assay Buffer prior to data acquisition. Samples were analysed using a FACSCalibur flow cytometer (BD Biosciences, Franklin Lakes, NJ, USA). To investigate the expression of CTLA-4 in MSCs, the ALDEFLUOR kit (Stemcell Technologies, Inc., Vancouver, BC, Canada) was used. The ALDEFLUOR ${ }^{\mathrm{TM}}$ reagent used the enzyme bodipy-aminoacetaldehyde (BAAA) as fluorescent substrate for ALDH, which freely diffused into intact and viable cells. BAAA was converted into a polar fluorescent product (BODIPY ${ }^{\mathrm{TM}}$-aminoacate) by ALDH and was retained inside the cells. Dead cells were excluded based on light scatter characteristics. A total of $1 \times 10^{6} / \mathrm{ml}$ cells were resuspended in an Assay Buffer (Stemcell Technologies, Inc., Vancouver, BC, Canada) at room temperature. A tube of cells was immediately quenched with $5 \mu \mathrm{l}$ specific inhibitor of the enzyme ALDH, with diethylaminobenzaldehyde (DEAB) as the negative control at room temperature. To all tubes, $5 \mu 1$ ALDEFLUOR $^{\mathrm{TM}}$ reagent was added and incubated for $45 \mathrm{~min}$ at $37^{\circ} \mathrm{C}$. In a number of experiments, cells were labelled with
CTLA-4 subsequent to being incubated with ALDEFLUOR. Data analysis was conducted using Cell Quest Pro (version 5.1; BD Biosciences).

Apoptosis detection. Viable and dead cells of B16-F0 and B16-F1 with or without anti-CTLA-4 antibody were assessed using double staining with fluorescein isothiocyanate (FITC)-annexin $\mathrm{V}$ and propidium iodide (BD Pharmingen; BD Biosciences) for $30 \mathrm{~min}$ at room temperature, following the manufacturer's protocol. Analysis was performed using flow cytometry, and the apoptotic percentages of annexin $\mathrm{V}^{+}$/propidium iodide ${ }^{-}$and annexin $\mathrm{V}^{+} /$propidium iodide ${ }^{+}$cells were calculated.

Tumoursphere culture. In tumoursphere culture, $1 \times 10^{6}$ cells of $\mathrm{B} 16-\mathrm{F} 0$ or B16-F1 were plated as single cells in ultralow attachment six-well plates (Corning, Lowell, MA, USA) without anti-CTLA-4 following the protocol of Duarte et al (20). Cells were briefly cultured for $24 \mathrm{~h}$ at $37^{\circ} \mathrm{C}$ in RPMI 1640 containing $6 \mathrm{mg} / \mathrm{ml}$ glucose (Sigma-Aldrich; Merck KGaA, Darmstadt, Germany), $1 \mathrm{mg} / \mathrm{ml} \mathrm{NaHCO}_{3}$ (Sigma-Aldrich; Merck KGaA), 5 mM 4-(2-hydroxyethyl)-1-piperazineethanesulfonic acid (Sigma-Aldrich; Merck KGaA), $4 \mu \mathrm{g} / \mathrm{ml}$ heparin (Sigma-Aldrich; Merck KGaA), $4 \mathrm{mg} / \mathrm{ml}$ bovine serum albumin (Sigma-Aldrich; Merck KGaA), 20 $\mathrm{pg} / \mathrm{ml}$ insulin (Sigma-Aldrich; Merck KGaA), and N2 supplement (Invitrogen; Thermo Fisher Scientific, Inc.) in addition to $10 \mathrm{ng} / \mathrm{ml}$ basic fibroblast growth factor (PeproTech, Inc., Rocky Hill, NJ, USA) and $20 \mathrm{ng} / \mathrm{ml}$ epidermal growth factor (PeproTech, Inc.). The second day following seeding, cells were treated with $10 \mu \mathrm{g}$ anti-CTLA-4 antibody (cat. no. 16-1521; 1:100; eBioscience; Thermo Fisher Scientific, Inc.) for 14 days at $37^{\circ} \mathrm{C}$. Tumourspheres were observed under a optical microscope (magnification, x40) 14 days later. Individual spheres $>100 \mu \mathrm{m}$ from each replicate well were counted under an inverted microscope.

Reverse transcription-quantitative polymerase chain reaction (RT-qPCR). B16-F0 and B16-F1 cells were cultured with or without anti-CTLA-4 antibody in RPMI-1640 for $48 \mathrm{~h}$ at $37^{\circ} \mathrm{C}$. RNAiso Plus ( $1 \mathrm{ml}$; Takara Bio, Inc., Otsu, Japan) was added to all the cultured B16-F0 and B16-F1 cells for total RNA extraction according to the manufacturer's protocol. cDNA was synthesized using the PrimeScriptRT Master Mix (Takara Bio, Inc.) following the manufacturer's protocol. The temperature protocol of reverse transcription was as follows: $37^{\circ} \mathrm{C}$ for $15 \mathrm{~min}, 85^{\circ} \mathrm{C}$ for $5 \mathrm{sec}$ and $4^{\circ} \mathrm{C}$ for $10 \mathrm{~min}$. A total of $20 \mathrm{ng}$ of resulting cDNA were subjected to RT-qPCR, in a $10 \mu \mathrm{l}$ final reaction volume and analysed in triplicates. The cycling conditions were as follows: $95^{\circ} \mathrm{C}$ for $30 \mathrm{sec}, 95^{\circ} \mathrm{C}$ for $5 \mathrm{sec} 40$ times and $60^{\circ} \mathrm{C}$ for $34 \mathrm{sec} 40$ times. Gene expression was detected using the ABI 7900 sequence detection system (Thermo Fisher Scientific, Inc.). The gene expression level of each target gene was normalized using the endogenous gene GAPDH and quantified using the real-time quantitative PCR and $2^{-\Delta \Delta \mathrm{Cq}}$ method (21). Relative value of sample $=2^{-(\Delta \mathrm{Cqinternal}}$ parameter- $\Delta$ Cqsample) . PCR primers were as follows: ALDH1A1 forward, 5'-GGCACTCAATGGTGGGAAAGTC-3' and reverse, 5'-ATGCCAGGTGAAGAGCCGTG-3'; ALDH1A3 forward, CCACGGTCTTCTCAGATGTTACG-3' and reverse, 5'-GCTGTGAGTCCATAGTCGGTGC-3'; ALDH2 forward, 

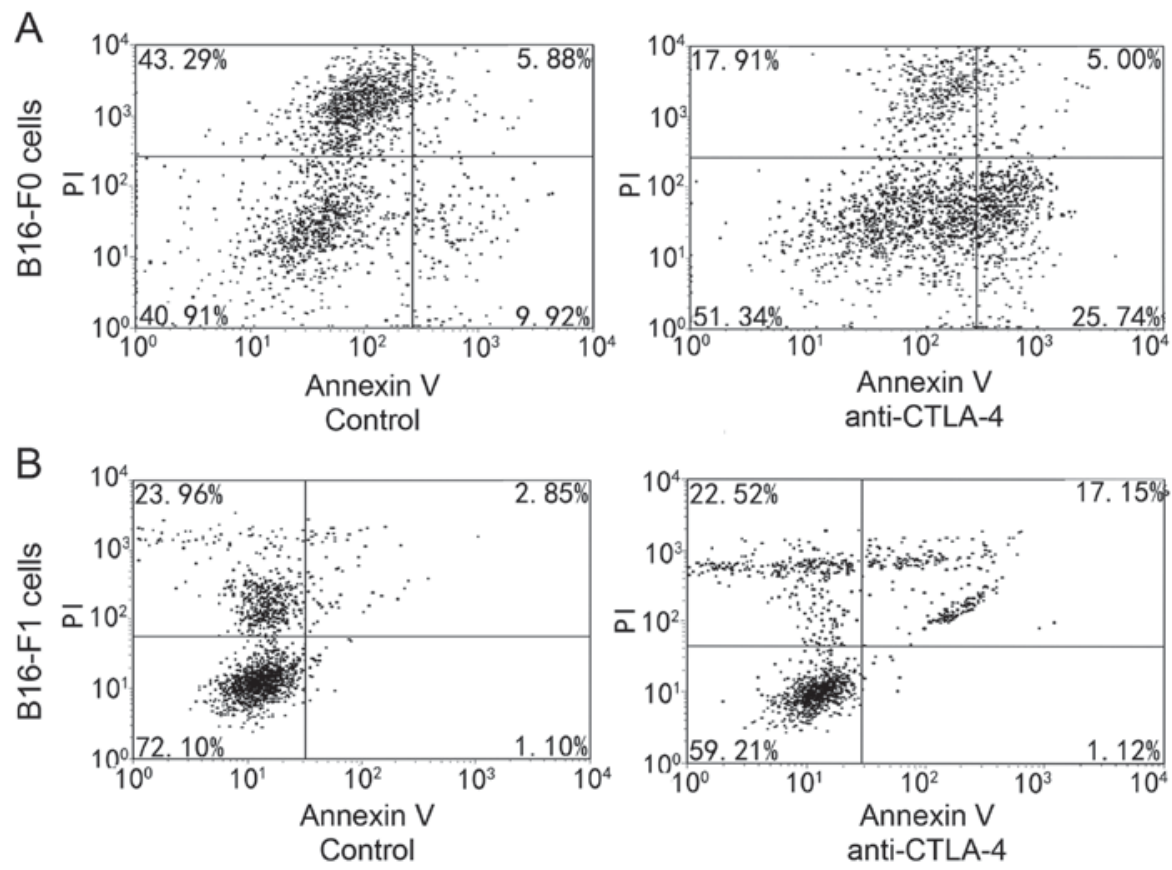

Figure 1. Melanoma cell-intrinsic CTLA-4 regulated cell apoptosis. Following the addition of 2,500 ng/ml anti-CLTA-4, the apoptosis rates of (A) B16-F0 cells and (B) B16-F1 cells were detected using flow cytometry. The results were expressed as the mean \pm standard error of the mean of five independent experiments. CTLA-4, cytotoxic T lymphocyte antigen-4.

5'-TTAACAATGAGTGGCACGACG-3' and reverse, 5'-CGC CAATCGGTACAACAGC-3'; and GAPDH forward, 5'-AGG AGCGAGACCCCACTAACA-3' and reverse, 5'-AGGGGG GCTAAGCAGTTGGT-3'. Baseline and threshold for the $2^{-\Delta \Delta \mathrm{Cq}}$ calculation were set automatically using Stepone plus 2.3 software. (Thermo Fisher Scientific, Inc., Waltham, MA, USA).

Animals and tumour model. For the in vivo experiments, 20 adult specific-pathogen-free C57BL/6 female mice $(22 \pm 4 \mathrm{~g})$ were provided by the Experimental Animal Center of Tongji Medical College of Huazhong University of Science and Technology. All mice were housed in the same facilities (temperature and humidity), consumed the same diet (Lab Diet) and water ad libitum, and were kept on an identical 12-h light/dark cycle. All animals were randomly assigned to two groups $(\mathrm{n}=10)$. The $\mathrm{C} 57$ mice were injected with $1 \times 10^{6} \mathrm{~B} 16-\mathrm{F} 0$ (F0 group) or B16-F1 (F1 group) tumour cells subcutaneously, and the tumour size was monitored with callipers every other day and sacrificed at the 28th day. Fourteen days after tumour cells injection, F0 or F1 group was injected intraperitoneally with anti-CTLA-4 antibody or PBS three times every other day. All surgical procedures and the handling of animals were performed in accordance with institutional guidelines. All procedures were approved by the Institutional Animal Care and Use Committee at Tongii Medical College of Huazhong University of Science and Technology (Hubei, China).

Statistical analysis. Statistical analyses were performed using Graph Pad Prism 5.0 software (GraphPad Software, Inc., La Jolla, CA, USA). All data were expressed as the mean \pm the standard error of the mean (SEM). Statistical analysis was performed using a two-tailed paired Student's t-test. $\mathrm{P}<0.05$ was considered to indicate a statistically significant difference.

\section{Results}

Melanoma cell-intrinsic CTLA-4 regulates cell apoptosis. Following a 48-h incubation with anti-CTLA-4, cells were harvested and annexin V/PI staining was assessed using flow cytometry. As presented in Fig. 1, exposure to 10,000 ng/ml anti-CLTA-4 was associated with marked apoptosis induction. Cells that were in early apoptosis were FITC-annexin $\mathrm{V}$-positive and PI-negative, and cells that were in late apoptosis were FITC-annexin V-positive and PI-positive. The rate of apoptosis meant the ratio of cells in early and late apoptosis to the total cell number. The rate of apoptosis increased by $14.68 \pm 4.11 \%$ in B16-F0 cells and to $15.92 \pm 2.42 \%$ in B16-F1 cells compared with the control.

Expression of CTLA-4 in MSCs. On the basis of the data from melanoma cells, the prevalence of CTLA-4 expression in MSCs was examined. One previous study reported that 5-10\% of B16-F0 and B16-F1 cells belonged to MSCs (22). $\mathrm{ALDH}^{+}$ cells were ALDEFLUOR positive and $\mathrm{ALDH}^{+} \mathrm{CTLA}-4^{+}$ cells were ALDEFLUOR positive and CTLA-4 positive. In the present study, $9.02 \pm 1.24 \%$ of the B16-F0 melanoma cells and $9.13 \pm 0.81 \%$ of B16-F1 melanoma cells were $\mathrm{ALDH}^{+}$cells (Fig. 2A) and $2-5 \%$ of the cells were $\mathrm{ALDH}^{+} \mathrm{CTLA}-4^{+}$cells (Fig. 2B). Of the $\mathrm{ALDH}^{+}$cells, 30-45\% expressed CTLA-4, indicating that CTLA-4 was mainly expressed in MSCs in melanoma cells.

CTLA-4 regulated the activity of ALDH in melanoma cells. To investigate the biological activity of CTLA-4 on MSCs, the effect of CTLA-4 suppression on the expression of ALDH1A1, ALDH1A3 and ALDH2 in B16-F0 and B16-F1 melanoma cells was examined. Fig. 3A reveals that anti-CTLA-4 significantly downregulated ALDH1A1 $(\mathrm{P}<0.01)$, ALDH1A3 $(\mathrm{P}<0.01)$ and 
A

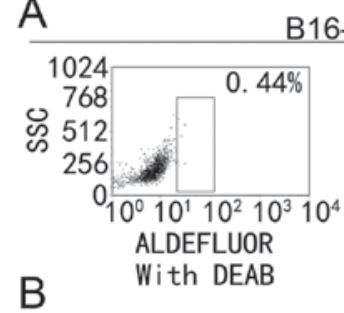

B16-F0 cells
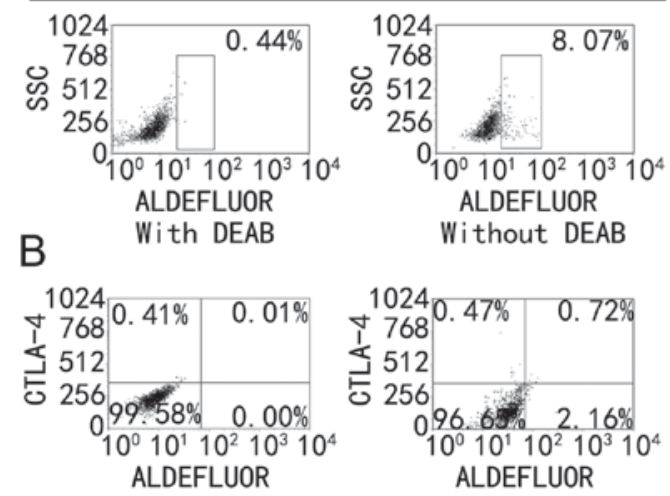
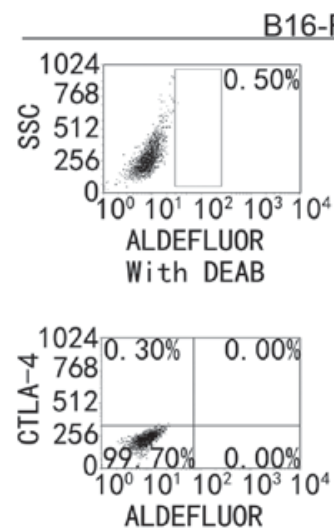

B16-F1 cells
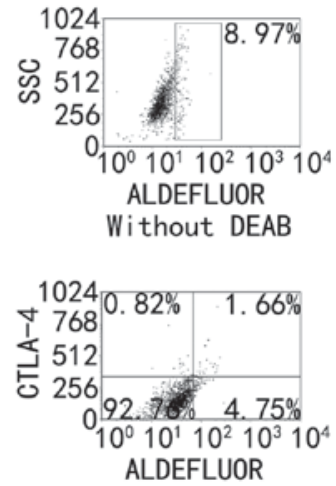

Figure 2. Expression of CTLA-4 in melanoma stem cells. (A) ALDH ${ }^{+}$cells were identified in two melanoma cell lines by flow cytometry with the ALDEFLUOR kit. (B) ALDH ${ }^{+}$CTLA-4 ${ }^{+}$cells were identified in two melanoma cell lines by flow cytometry. CTLA-4, cytotoxic T lymphocyte antigen-4; ALDH, aldehyde dehydrogenase; DEAB, diethylaminobenzaldehyde.

A
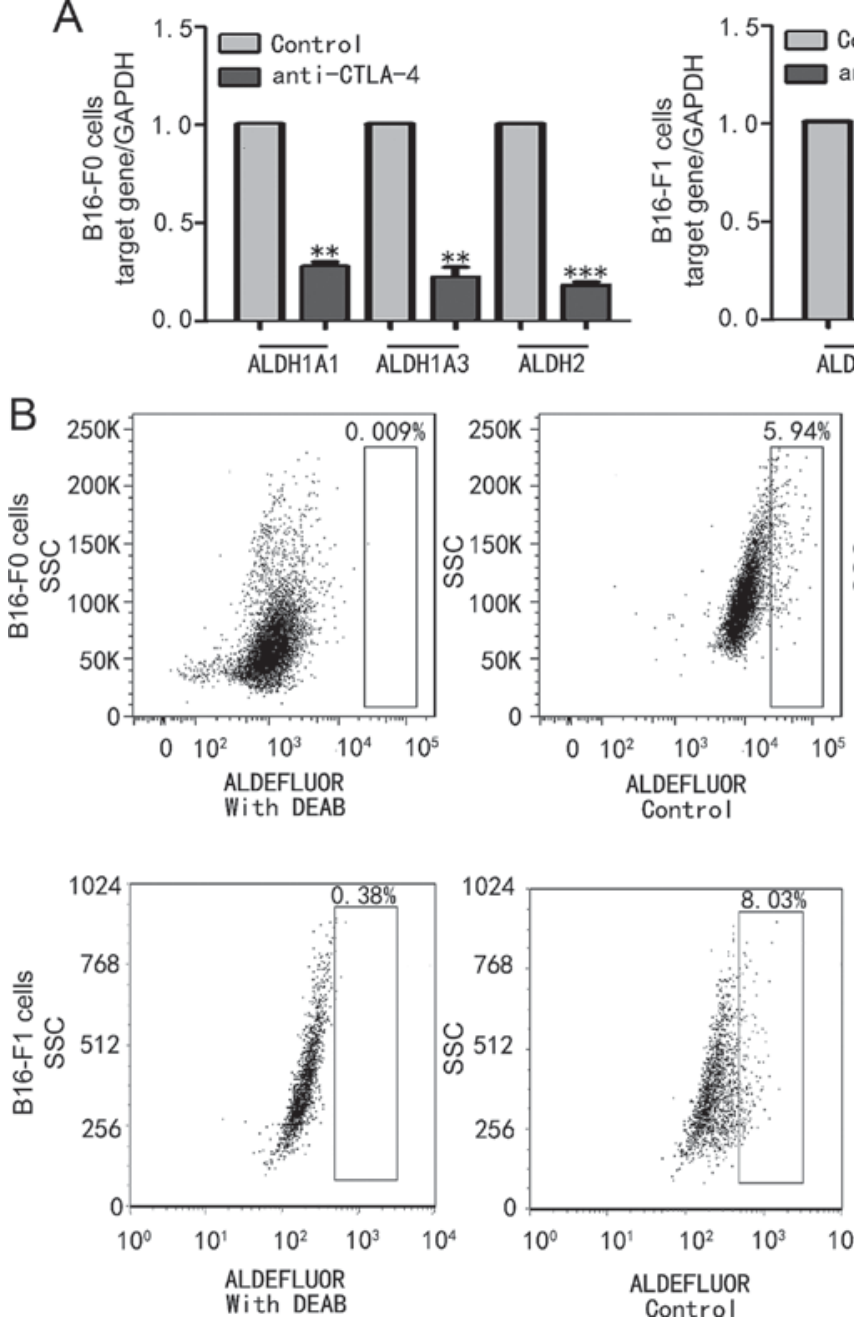
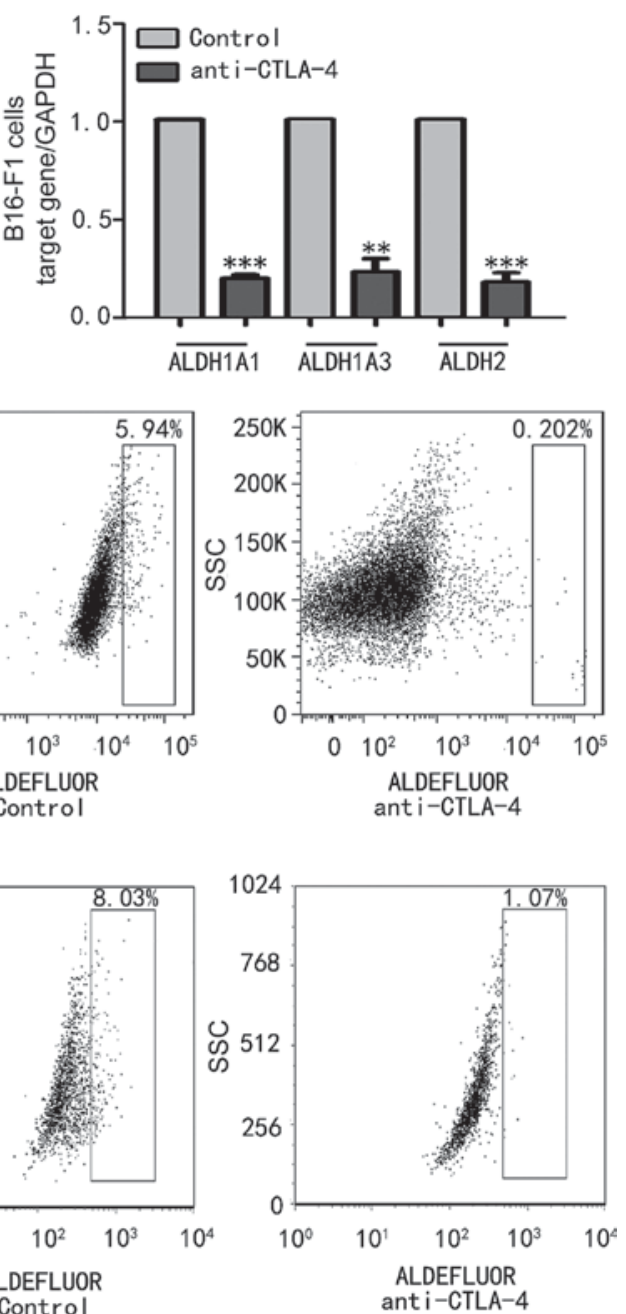

Figure 3. CTLA-4 regulates the activity of ALDH in melanoma cells. (A) Downregulation of ALDH1A1, ALDH1A3 and ALDH2 mRNA by anti-CTLA-4 in B16-F0 and B16-F1 cells was revealed using reverse transcription-quantitative polymerase chain reaction analysis, using GAPDH as the internal standard. ALDH1A1, ALDH1A3 and ALDH2 mRNA expression levels were quantified and normalized to the amplified GAPDH products. Each assay was repeated three times. ${ }^{* *} \mathrm{P}<0.01$ and ${ }^{* * *} \mathrm{P}<0.001$ vs. the control. (B) Regulation of CTLA-4 on the expression of ALDH protein in B16-F0 and B16-F1 cells was detected by flow cytometry. CTLA-4, cytotoxic T lymphocyte antigen-4; ALDH, aldehyde dehydrogenase; DEAB, diethylaminobenzaldehyde.

ALDH2 $(\mathrm{P}<0.001)$ mRNA expression levels compared with their respective controls in B16-F0 cells, and significantly downregulated ALDH1A1 $(\mathrm{P}<0.001)$, ALDH1A3 $(\mathrm{P}<0.01)$ and ALDH2 $(\mathrm{P}<0.001)$ mRNA expression levels compared with their respective controls in B16-F1 cells. The expression levels of ALDH1A1, ALDH1A3 and ALDH2 mRNA were 

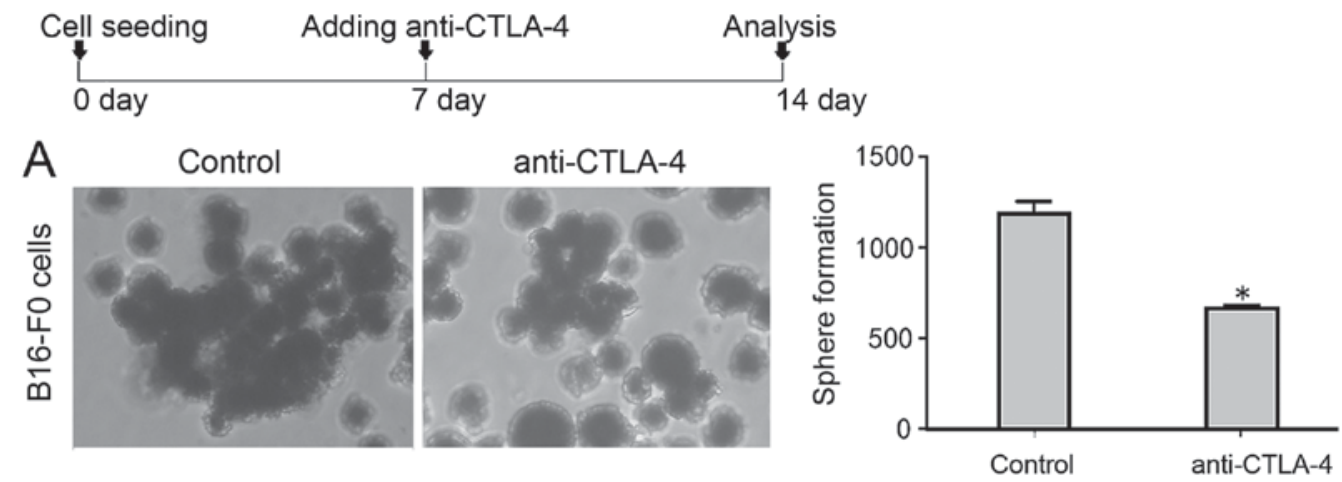

\section{B}
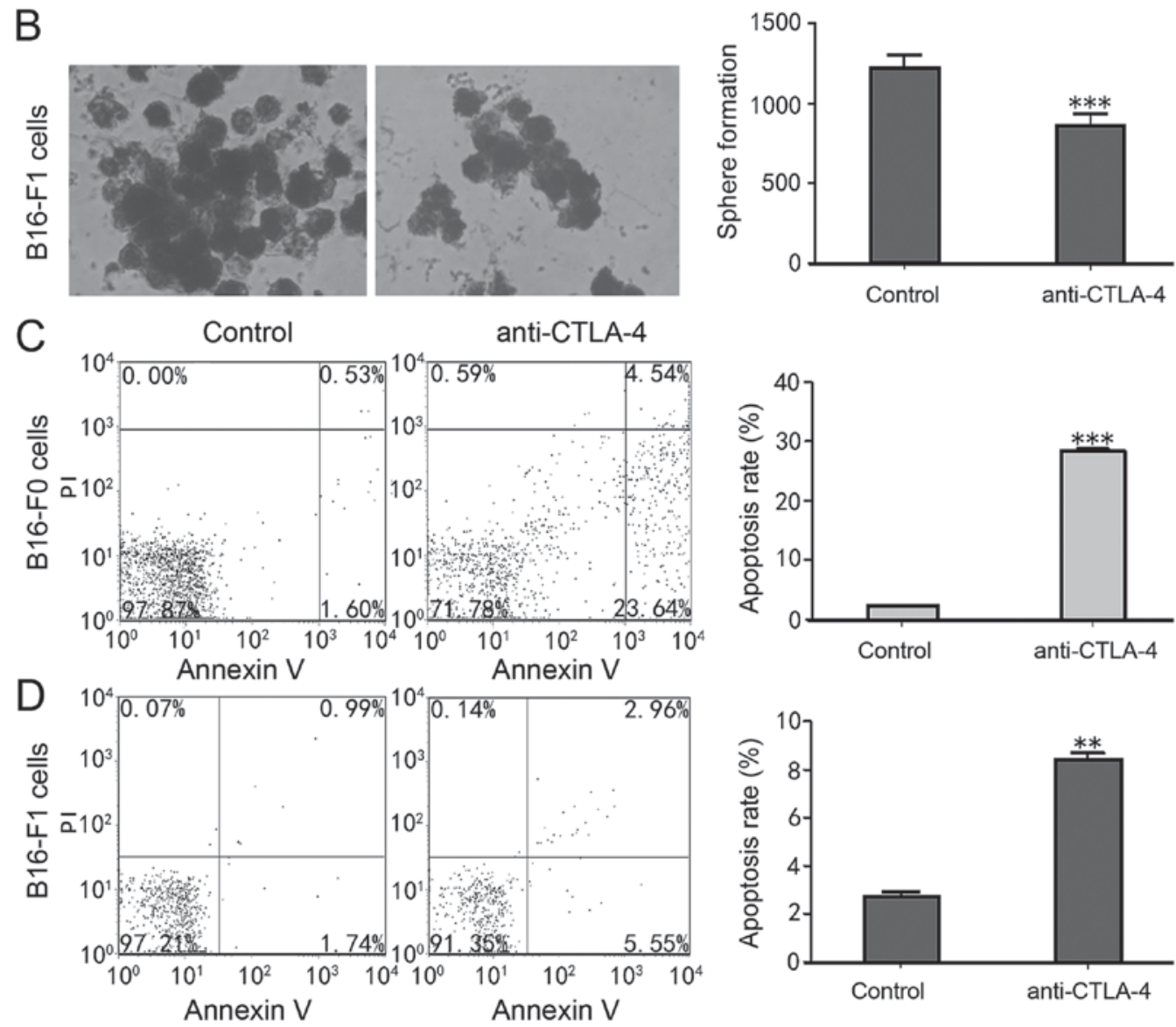

Figure 4. CTLA-4 inhibited sphere formation and induced the apoptosis of sphere cells. Following the culturing with anti-CTLA-4, the abilities of sphere formation of (A) B16-F0 cells and (B) B16-F1 cells were significantly reduced under an optical microscope (magnification, $\mathrm{x} 40$ ). Following the addition of $10,000 \mathrm{ng} / \mathrm{ml}$ anti-CLTA-4 antibody, the apoptosis rates of (C) B16-F0 spheres and (D) B16-F1 spheres were detected using flow cytometry. ${ }^{*} \mathrm{P}<0.05,{ }^{* *} \mathrm{P}<0.01$ and ${ }^{* * *} \mathrm{P}<0.001$ vs. the control. CTLA-4, cytotoxic T lymphocyte antigen-4; ALDH, aldehyde dehydrogenase.

inhibited by $\sim 80 \%$ in B16-F0 and B16-F1 melanoma cells (Fig. 3A). These results were confirmed by flow cytometry. Once the cells were treated with anti-CTLA-4, the expression of ALDH was decreased to $0.202 \%$ (Fig. 3B), and the inhibition rate of ALDH expression in B16-F0 and B16-F1 cells was $81.12 \pm 5.842$ and $79.35 \pm 4.438 \%$, respectively. These results indicate that CTLA-4 is involved in the regulation of ALDH activity and affects the biological behaviour of MSCs.

CTLA-4 affected sphere formation by melanoma cells. To assess whether CTLA-4 expression affects the self-renewal of MSCs, melanoma cell lines were cultured with anti-CTLA-4. It was revealed that anti-CTLA-4 significantly reduced the tumoursphere-forming efficiency of B16-F0 and
B16-F1 melanoma cells compared with the control groups (Fig. 4A and B). Cancer stem cells may be serially passaged to generate secondary spheres with a cellular composition resembling that of the primary sphere (23). The secondary tumourspheres generated from the anti-CTLA-4 cultured melanoma cell-derived tumourspheres were significantly decreased, by $\sim 2$-fold in B16-F0 $(\mathrm{P}<0.05)$ and $\sim 1.4$-fold in $\mathrm{B} 16-\mathrm{F} 1 \quad(\mathrm{P}<0.001)$ melanoma cells in number and size, compared with the control groups (Fig. 4A and B). The effect of anti-CTLA-4 on melanoma cancer stem-like cell apoptosis was detected. The results revealed that anti-CTLA-4 significantly increased the rate of apoptosis in B16-F0 $(\mathrm{P}<0.001)$ and $\mathrm{B} 16-\mathrm{F} 1(\mathrm{P}<0.01)$ cells, and resulted in a $4-15$-fold increase in the rate of apoptosis in melanoma cancer stem-like cells (from 


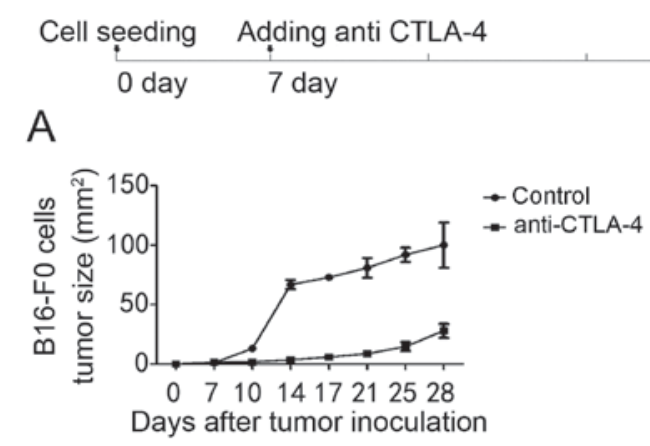

Analysis

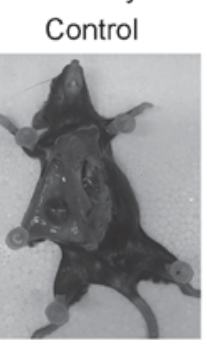

$\mathrm{B}$
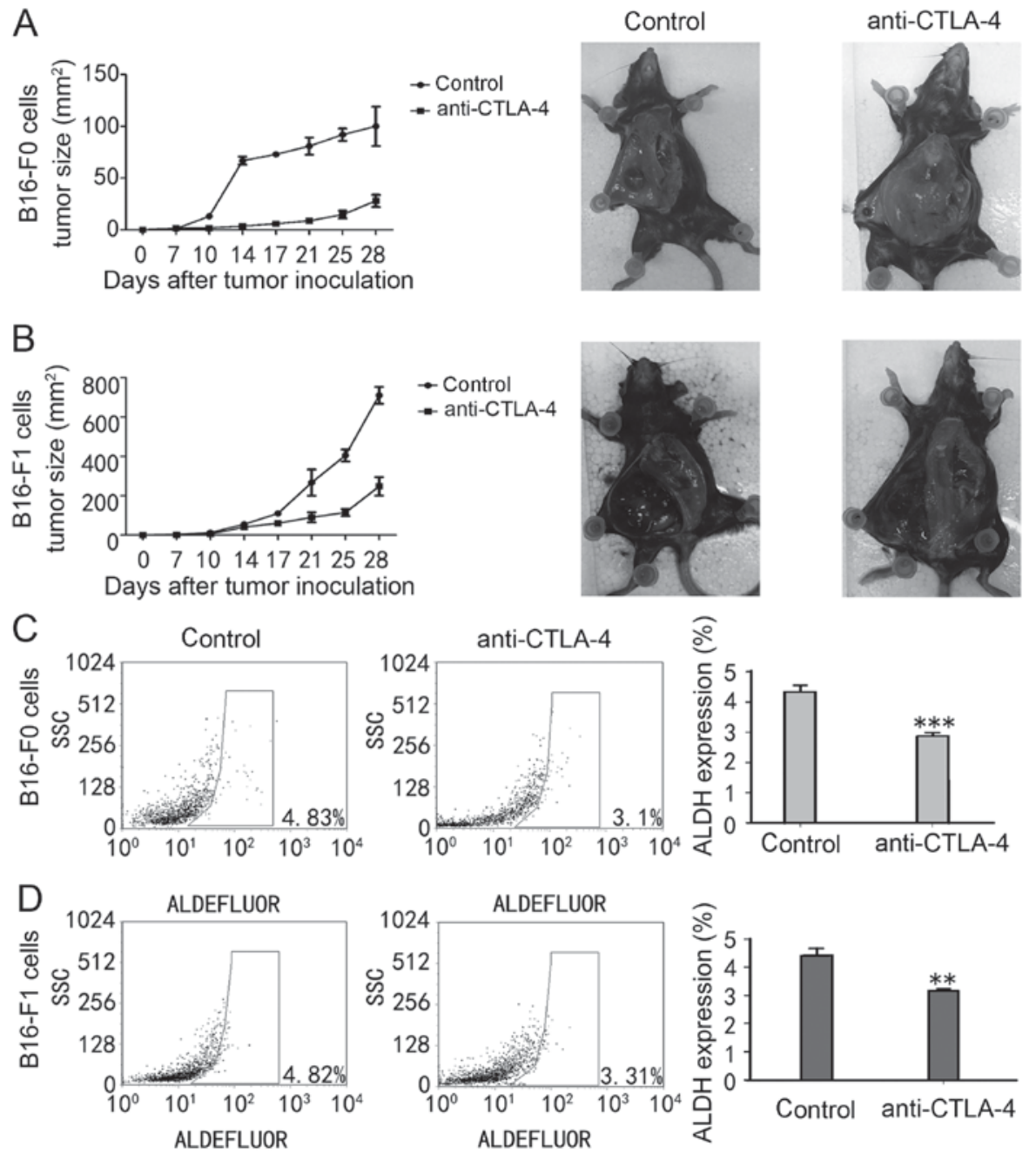

Figure 5. CTLA-4 affected the tumourigenic capacity of melanoma cells. C57BL/6 mice were inoculated with (A) B16-F0 cells and (B) B16-F1 cells for 7 days, separately. C57BL/6 mice were then administered with CTLA- 4 inhibitor. The tumour size of the melanoma was monitored. The residual ALDH ${ }^{+}$ MSCs within the tumour were analysed by flow cytometry in a (C) B16-F0 mouse model and (D) B16-F1 mouse model. ${ }^{* *} \mathrm{P}<0.01$ and ${ }^{* * *} \mathrm{P}<0.001$ vs. the control. CTLA-4, cytotoxic T lymphocyte antigen-4; ALDH, aldehyde dehydrogenase.

$28.32 \pm 0.43$ to $2.19 \pm 0.83 \%$ in B16-F0 cells and from $8.42 \pm 0.27$ to $8.42 \pm 0.27 \%$ in B16-F1 cells; Fig. $4 \mathrm{C}$ and D). Therefore, these results indicate that CTLA-4 promoted the tumoursphere forming capacity of MSCs and inhibited their apoptosis.

CTLA-4 affected the tumourigenic capacity of melanoma cells. To confirm the function of CTLA-4 in the self-renewal capacity of MSCs, whether CTLA-4 affected the tumourigenic capacity of melanoma cells in vivo was evaluated (Fig. 5A and B). Compared with PBS-injected mice, anti-CTLA-4 suppressed tumour growth in two mice models, which is supported by previous observations (24). In addition, residual $\mathrm{ALDH}^{+} \mathrm{MSCs}$ were observed within the tumour. As presented in Fig. 5C and D, anti-CTLA-4 significant decreased the residual $\mathrm{ALDH}^{+} \mathrm{MSCs}$ in the two mice models compared with PBS-injected animals (from 4.35 \pm 0.22 to $2.85 \pm 0.13 \%$ in B16-F0-injected mice models, $\mathrm{P}<0.001$; from $4.41 \pm 0.25$ to $3.15 \pm 0.28 \%$ in B16-F1-injected mice models, $\mathrm{P}<0.01)$. These results depict a potential mechanism for the anti-tumour effects of anti-CTLA-4 associated with its ability to suppress the tumourigenic capacity of MSCs.

\section{Discussion}

Extensive clinical evidence supports that CTLA-4 targeting agents demonstrate a notable benefit in patients with advanced melanoma due to the function of CTLA-4 in negatively regulating $\mathrm{T}$ cell activation $(8,9)$. In addition to T cells, CTLA-4 is expressed on cell lines derived from a variety of human malignant solid tumour types, including melanoma, neuroblastoma, oesophageal carcinoma, lung cancer, gastric and colorectal cancer types $(13,15,17,18)$. CTLA-4 was revealed to serve a function in the protection against apoptosis (18). These findings are consistent with the results from a study by Contardi et al (18) regarding the association between CTLA-4 expression in tumour cells and tumour cell apoptosis. These studies indicate the function of the CTLA-4 molecule in tumour cells and the notable function of CTLA-4 in stem cells. Dai et al (19) reported that CTLA-4 was over expressed in allogeneic mesenchymal stem cells and promoted osteogenic differentiation. Shinoyama et al (25) revealed that CTLA-4 promoted neuronal differentiation in the grafts of embryonic stem cell-derived 
neural precursor cells. These findings confirmed that CTLA-4 regulated the differentiation of stem cells.

Studies have demonstrated that MSCs drive the initiation and progression of a melanoma based on the capacity for self-renewal, multiple differentiation, chemoresistance and immune evasion $(2,3)$. Thus, targeting the survival mechanisms utilized by MSCs may be the most effective way to cure melanoma. Previous studies have successfully used ALDEFLUOR/ALDH as a marker to isolate MSCs from mice $(22,26)$. However, the mechanisms underlying the biological effects in $\mathrm{ALDH}^{+} \mathrm{MSCs}$ remain unclear. In the present study, it was revealed that CTLA-4 was expressed in $\mathrm{ALDH}^{+} \mathrm{MSC}$, in which it regulated the activity of ALDH in melanoma cells. These results indicated that CTLA-4 induced melanoma progression and may contribute to its function in regulating the stemness of MSCs.

To elucidate the function of CTLA-4 signalling pathways in $\mathrm{ALDH}^{+} \mathrm{MSCs}$ further, the effect of anti-CTLA-4 on tumourigenic capacity was examined. The results of the present study demonstrated, to the best of our knowledge for the first time, that anti-CTLA-4 may efficiently inhibit the capacity for tumoursphere formation and induce apoptosis in melanoma cancer stem-like cells in vitro. In vivo, the results demonstrated that treatment with anti-CTLA-4 suppressed tumour growth and significantly decreased residual $\mathrm{ALDH}^{+} \mathrm{MSCs}$. These results indicate that the blockage of CTLA-4 may target $\mathrm{ALDH}^{+} \mathrm{MSCs}$, which is a novel non-immunological mechanism for the anti-tumour activity of anti-CTLA-4. Ipilimumab (an anti-CTLA-4 monoclonal antibody) confers a long-term survival benefit in $\sim 20 \%$ of patients with advanced melanoma in an unselected population (27), and identifying those patients may prove to be valuable. One previous study revealed that immune activation in responding patients increased the absolute lymphocyte count and upregulated the T-cell activation marker inducible T-cell co-stimulator and the tumour antigen New York oesophageal squamous cell carcinoma 1 (28). The tumour mutation volume and high baseline expression levels of immune-associated genes, including C-C motif chemokine receptor 2 (CCR2), CCR-like 2 and CCR5, additionally aid prediction of the activity of immune checkpoint blockade (29). In fact, no predictive biomarkers for the selection of patients with melanoma able to receive ipilimumab are sufficiently robust enough for clinical use. However, previous studies have illustrated that, in breast and oesophageal cancer, CTLA-4 expression on tumour cells was associated with a poor prognosis $(6,12)$. According to the biological characteristics of MSCs, the expression of CTLA-4 on tumour cells, indicating poor prognosis, may be associated with the regulation of CTLA-4 on cancer stem cells. The present study has revealed that the regulation of CTLA-4 on MSCs may serve as a predictive biomarker for selecting patients with melanoma to receive anti-CTLA-4 treatment.

In conclusion, the present findings illustrated that melanoma cell-intrinsic CTLA-4 induced tumourigenesis and inhibited apoptosis, in addition to its pro-tumourigenic function. Identifying melanoma-CTLA-4-driven tumourigenesis critically improves the current understanding of the mechanism underlying melanoma progression and may benefit CTLA-4-targeted therapy by improving the long-term outcomes in patients with advanced-stage melanoma.

\section{Acknowledgements}

The authors would like to thank the Center for Stem Cell Research and Application for providing flow analysis and Dr Wen Lin (Department of Pediatrics, Affiliated Union Hospital, Wuhan, China) for their insights.

\section{Funding}

The present study was supported by the National Nature Sciences Foundation of China (grant no. 81301954) and the Hubei Provincial Health and Family Planning Scientific Research Project (grant no. 2015060101010043).

\section{Availability of data and materials}

The datasets used and/or analysed during the current study are available from the corresponding author on reasonable request.

\section{Authors' contributions}

BZ performed the flow analysis and analysed all data, and was a major contributor in writing the manuscript. FZ helped to design the stufy, performed the flow analysis and was a important contributor in writing the manuscript. JD cultured the cells. DB made the animal model. CW performed the western blot. JH performed the RT-qPCR. All authors read and approved the final manuscript.

\section{Ethics approval and consent to participate}

The present study was approved by the Institutional Animal Care and Use Committee at Tongii Medical College of Huazhong University of Science and Technology (Hubei, China).

\section{Patient consent for approval}

Not applicable.

\section{Competing interests}

The authors declare that they have no competing interests.

\section{References}

1. Gray-Schopfer V, Wellbrock C and Marais R: Melanoma biology and new targeted therapy. Nature 445: 851-857, 2007.

2. Murphy GF, Wilson BJ, Girouard SD, Frank NY and Frank MH: Stem cells and targeted approaches to melanoma cure. Mol Aspects Med 39: 33-49, 2014.

3. Hirohashi Y, Torigoe T, Tsukahara T, Kanaseki T, Kochin V and Sato N: Immune responses to human cancer stem-like cells/cancer-initiating cells. Cancer Sci 107: 12-17, 2016.

4. Brunner MC, Chambers CA, Chan FK, Hanke J, Winoto A and Allison JP: CTLA-4-mediated inhibition of early events of T cell proliferation. J Immunol 162: 5813-5820, 1999.

5. Wu L, Yun Z, Tagawa T, Rey-McIntyre K and de Perrot M: CTLA-4 blockade expands infiltrating T cells and inhibits cancer cell repopulation during the intervals of chemotherapy in murine mesothelioma. Mol Cancer Ther 11: 1809-1819, 2012.

6. Whiteside TL: Regulatory T cell subsets in human cancer: Are they regulating for or against tumor progression? Cancer Immunol Immunother: 63: 67-72, 2014. 
7. Lindau D, Gielen P, Kroesen M, Wesseling P and Adema GJ The immunosuppressive tumour network: Myeloid-derived suppressor cells, regulatory $\mathrm{T}$ cells and natural killer $\mathrm{T}$ cells. Immunology 138: 105-115, 2013.

8. Hodi FS, O'Day SJ, McDermott DF, Weber RW, Sosman JA, Haanen JB, Gonzalez R, Robert C, Schadendorf D, Hassel JC, et al: Improved survival with ipilimumab in patients with metastatic melanoma. N Engl J Med 363: 711-723, 2010

9. Robert C, Thomas L, Bondarenko I, O'Day S, Weber J, Garbe C, Lebbe C, Baurain JF, Testori A, Grob JJ, et al: Ipilimumab plus dacarbazine for previously untreated metastatic melanoma. N Engl J Med 364: 2517-2526, 2011.

10. Suwalska K, Pawlak E, Karabon L, Tomkiewicz A, Dobosz T, Urbaniak-Kujda D, Kuliczkowski K, Wolowiec D, Jedynak A and Frydecka I: Association studies of CTLA-4, CD28 and ICOS gene polymorphisms with B-cell chronic lymphocytic leukemia in the Polish population. Hum Immunol 69: 193-201, 2008.

11. Monne M, Piras G, Palmas A, Arru L, Murineddu M, Latte G, Noli A and Gabbas A: Cytotoxic T-lymphocyte antigen-4 (CTLA-4) gene polymorphism and susceptibility to non-Hodgkin's lymphoma. Am J Hematol 76: 14-18, 2004.

12. Yu H, Yang J, Jiao S, Li Y, Zhang W and Wang J: Cytotoxic $\mathrm{T}$ lymphocyte antigen 4 expression in human breast cancer: Implications for prognosis. Cancer Immunol Immunother 64: 853-860, 2015.

13. Khaghanzadeh N, Erfani N, Ghayumi MA and Ghaderi A CTLA4 gene variations and haplotypes in patients with lung cancer. Cancer Genet Cytogenet 196: 171-174, 2010

14. Laurent S, Queirolo P, Boero S, Salvi S, Piccioli P, Boccardo S, Minghelli S, Morabito A, Fontana V, Pietra G, et al: The engagement of CTLA-4 on primary melanoma cell lines induces antibody-dependent cellular cytotoxicity and TNF-alpha production. J Transl Med 11: 108, 2013.

15. Hadinia A, Hossieni SV, Erfani N, Saberi-Firozi M, Fattahi MJ and Ghaderi A: CTLA-4 gene promoter and exon 1 polymorphisms in Iranian patients with gastric and colorectal cancers. J Gastroenterol Hepatol 22: 2283-2287, 2007.

16. Pawlak E, Karabon L, Wlodarska-Polinska I, Jedynak A, Jonkisz A, Tomkiewicz A, Kornafel J, Stepien M, Ignatowicz A, Lebioda A, et al: Influence of CTLA-4/CD28/ICOS gene polymorphisms on the susceptibility to cervical squamous cell carcinoma and stage of differentiation in the Polish population. Hum Immunol 71: 195-200, 2010.

17. Zhang XF, Pan K, Weng DS, Chen CL, Wang QJ, Zhao JJ, Pan QZ, Liu Q, Jiang SS, Li YQ, et al: Cytotoxic T lymphocyte antigen-4 expression in esophageal carcinoma: Implications for prognosis. Oncotarget 7: 26670-26679, 2016.

18. Contardi E, Palmisano GL, Tazzari PL, Martelli AM, Falà F, Fabbi M, Kato T, Lucarelli E, Donati D, Polito L, et al: CTLA-4 is constitutively expressed on tumor cells and can trigger apoptosis upon ligand interaction. Int J Cancer 117: 538-550, 2005.
19. Dai F, Zhang F, Sun D, Zhang ZH, Dong SW and Xu JZ: CTLA4 enhances the osteogenic differentiation of allogeneic human mesenchymal stem cells in a model of immune activation. Braz $\mathrm{J}$ Med Biol Res 48: 629-636, 2015.

20. Duarte S, Momier D, Baqué P, Casanova V, Loubat A, Samson M, Guigonis JM, Staccini P, Saint-Paul MC, De Lima MP, et al: Preventive cancer stem cell-based vaccination reduces liver metastasis development in a rat colon carcinoma syngeneic model. Stem Cells 31: 423-432, 2013.

21. Livak KJ and Schmittgen TD: Analysis of relative gene expression data using real-time quantitative PCR and the 2(-Delta Delta C (T)) method. Methods 25: 402-408, 2001.

22. Ning N, Pan Q, Zheng F, Teitz-Tennenbaum S, Egenti M, Yet J, Li M, Ginestier C, Wicha MS, Moyer JS, et al: Cancer stem cell vaccination confers significant antitumor immunity. Cancer Res 72: 1853-1864, 2012.

23. Choi AR, Park JR, Kim RJ, Kim SR, Cho SD, Jung JY and Nam JS: Inhibition of Wnt1 expression reduces the enrichment of cancer stem cells in a mouse model of breast cancer. Biochem Biophys Res Commun 425: 436-442, 2012.

24. Li F, Guo Z, Yu H, Zhang X, Si T, Liu C, Yang X and Qi L: Anti-tumor immunological response induced by cryoablation and anti-CTLA-4 antibody in an in vivo RM-1 cell prostate cancer murine model. Neoplasma 61: 659-671, 2014.

25. Shinoyama M, Ideguchi M, Hayashi H, Doi D, Hashimoto N, Suzuki $\mathrm{M}$ and Takahashi J: Cytotoxic T lymphocyte antigen 4 immunogloblin promotes neuronal differentiation in the grafts of embryonic stem cell-derived neural precursor cells. Neuroscience 202: 484-491, 2012.

26. Luo Y, Dallaglio K, Chen Y, Robinson WA, Robinson SE, McCarter MD, Wang J, Gonzalez R, Thompson DC, Norris DA, et al: ALDH1A isozymes are markers of human melanoma stem cells and potential therapeutic targets. Stem Cells 30: 2100-2113, 2012.

27. Topalian SL, Taube JM, Anders RA and Pardoll DM: Mechanism-driven biomarkers to guide immune checkpoint blockade in cancer therapy. Nat Rev Cancer 16: 275-287, 2016.

28. Callahan MK, Postow MA and Wolchok JD: Immunomodulatory therapy for melanoma: Ipilimumab and beyond. Clin Dermatol 31: 191-199, 2013.

29. Ji RR, Chasalow SD, Wang L, Hamid O, Schmidt H, Cogswell J, Alaparthy S, Berman D, Jure-Kunkel M, Siemers NO, et al: An immune-active tumor microenvironment favors clinical response to ipilimumab. Cancer Immunol Immunother 61: 1019-1031, 2012. 